\title{
Development of annular dark field confocal scanning transmission electron microscopy
}

\author{
M. Takeguchi ${ }^{*}$, A. Hashimoto ${ }^{* *}$, K. Mitsuishi ${ }^{* * *}$, M. Shimojo $^{* * * *}$ \\ * Nano-characterization Center, National Institute for Materials Science, 3-13 Sakura, \\ Tsukuba 305-003, Japan \\ ** International Center for Young Scientists, National Institute for Materials Science, \\ 1-2-1 Sengen, Tsukuba 305-0047, Japan \\ *** Quantum Dot Research Center, National Institute for Materials Science, 3-13 Sakura, \\ Tsukuba 305-003, Japan \\ ${ }^{* * * *}$ Advanced Science Research Laboratory, Saitama Institute of Technology, 1690 \\ Fusaiji, Fukuya, Saitama 369-0293, Japan
}

Confocal imaging in scanning transmission electron microscopy (STEM) is a new technique for performing depth sectioning of specimens in a transmission electron microscope. Zaluzec et al. demonstrated confocal imaging in a conventional STEM machine for the first time and succeeded in observation inner structures of thick specimens with high amplitude contrast [1]. However, bright field mode confocal STEM that use a direct beam passing through a specimen is not capable of depth slicing enough to perform three-dimensional (3D) reconstruction. Phase contrast in coherent confocal imaging, which is thought to have high transverse resolution, is very low and can hardly produce interpretable 3D images [2-4].

In the present work, annular dark field (ADF) mode confocal STEM technique is proposed to improve depth resolution. An ADF aperture is fabricated with a focused ion beam machine and inserted into the objective aperture position of a conventional transmission electron microscope, JEM-2100F $(\mathrm{Cs}=1.0 \mathrm{~mm})$, equipped with a specimen scanning system and a pinhole [5]. We fix a finely focused beam (convergence semi-angle $10 \mathrm{mrad}$ ) on the specimen and scan the specimen instead of scanning the beam so that STEM images are obtained. For confocal imaging, the focused incident beam is magnified and projected on the pinhole plane by imaging lenses. The ADF aperture cut off a direct beam and only scattered electrons are focused on the pinhole plane as shown in Fig.1. Electrons coming from a specific depth in the specimen hit the detector through the pinhole and most of the other electrons from out-of-focus depths are rejected by the pinhole. Thus ADF confocal STEM is performed.

Figure 2 shows ADF confocal STEM images of polymerized carbon with cobalt nanoparticles on a carbon film taken at different $z$-positions (the $z$-difference is 100 $\mathrm{nm})$. The scanning speed was $0.1 \mathrm{~ms} /$ point, the image size was $512 \times 512$ pixels $^{2}$, and the acquisition time was $45 \mathrm{~s}$. It is shown that different objects exhibit bright contrast between two images. This means that objects at the specific depth can have bright contrast in the ADF confocal STEM and its depth resolution is approximately $100 \mathrm{~nm}$, while the remarkable contrast difference could not be seen in the same area at the same 
magnification in conventional ADF STEM observations even if the focus was changed by $1000 \mathrm{~nm}$.

\section{References}

[1] N. J. Zaluzec, Microscopy- Today 6 (2003) 8.

[2] J. J. Einspahr and P. M. Voyles, Ultramicrosc. 106 (2006) 1041.

[3] E. C. Cosgriff et al., Ultramicrosc. 108 (2008) 1558.

[4] K. Mitsuishi et al., Ultramicrosc. 108 (2008) 981.

[5] M. Takeguchi et al., J. Electron Microsc. 58 (2008) 123.

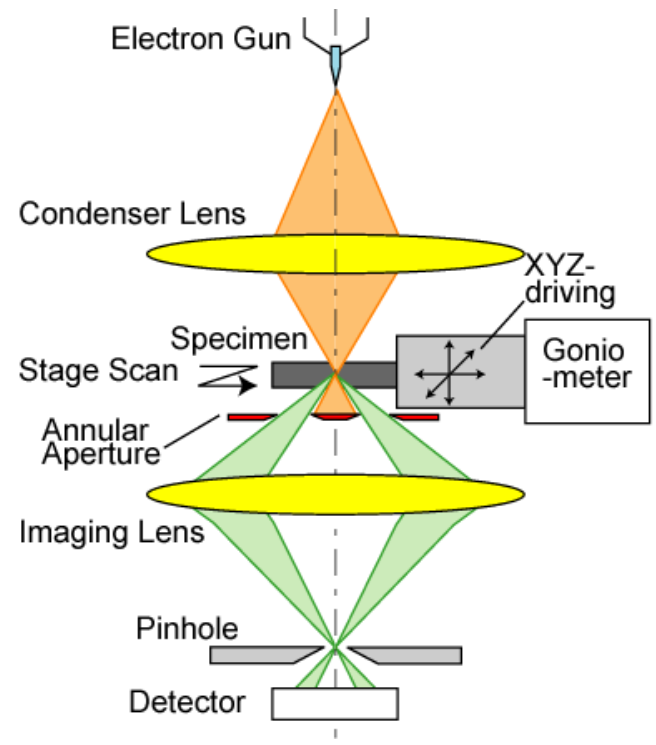

FIG.1 Schematic diagram of an ADF confocal STEM with a stage-scanning system. The specimen is three-dimensionally scanned with a fixed electron optics configuration.
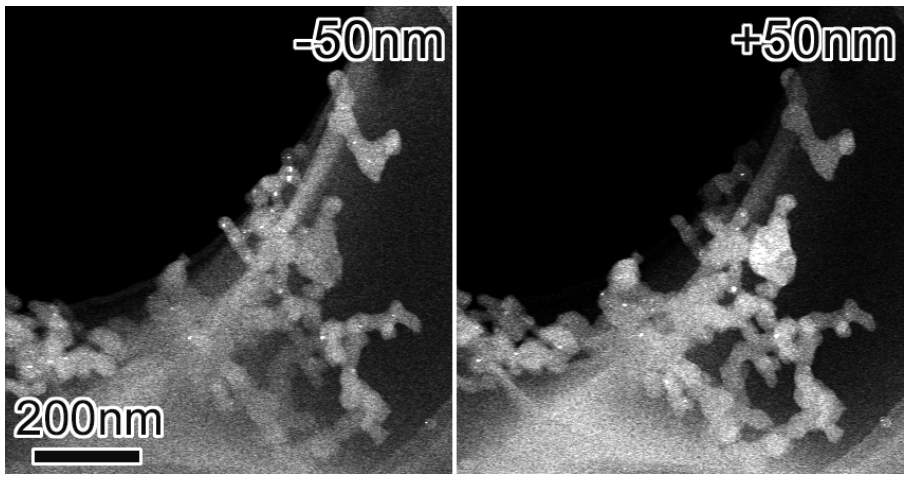

FIG.2 ADF confocal STEM images taken at $100 \mathrm{~nm}$ different z-positions (Sample : polymerized carbon with cobalt nanoparticles on a carbon film). 